EGU2020-16003

https://doi.org/10.5194/egusphere-egu2020-16003

EGU General Assembly 2020

(c) Author(s) 2020. This work is distributed under

the Creative Commons Attribution 4.0 License.

\title{
Wave-resonance fingerprint in the 2010 summer: a modelling experiment
}

\author{
Giorgia Di Capua ${ }^{1,2}$, Kai Kornhuber $^{3}$, Eftychia Rousi ${ }^{1}$, Sarah Sparrow ${ }^{4}$, David Wallom ${ }^{4}$, and Dim \\ Coumou ${ }^{1,2}$ \\ ${ }^{1}$ Potsdam Institute for Climate Impact Research, Potsdam, Germany (dicapua@pik-potsdam.de) \\ ${ }^{2}$ IVM-Institute for Environmental Studies, Free University of Amsterdam, Amsterdam, Netherlands \\ ${ }^{3}$ Earth Institute, Columbia University, New York, United States \\ ${ }^{4}$ Oxford e-Research Centre, University of Oxford, Oxford, United Kingdom
}

Summer 2010 was characterized by two contemporaneous extreme events: the Russian heat wave and the Pakistan flood. Several studies have shown a link between the two events, and QuasiResonant Amplification (QRA) has been suggested as an atmosphere-dynamic mechanism leading to the anomalous wavy circulation pattern which connected both extremes. Here, we aim at reproducing the 2010 circulation conditions in the Northern Hemisphere by obtaining a large ensemble of simulations from the Weather@home project within climateprediction.net (CPDN). We identify those ensemble members exhibiting a specific latitudinal temperature profile characterised by amplified high-latitude land warming (QRA - fingerprint) and investigate their surface temperature and upper level circulation properties. We show that when the QRA fingerprint is present, the mid-latitude circulation bears similar characteristics to those observed in the 2010 summer: hot temperatures over European Russia and a wavy pattern in the uppertropospheric meridional winds. As temperature profiles are projected to become increasingly similar to the QRA-fingerprint under future emission scenarios, these results provide further evidence that high latitude warming might favour persistent surface weather in the mid-latitudes. 